\title{
PICK INTERPOLATION IN SEVERAL VARIABLES
}

\author{
RYAN HAMILTON \\ (Communicated by Richard Rochberg)
}

Abstract. We investigate the Pick problem for the polydisk and unit ball using dual algebra techniques. Some factorization results for Bergman spaces are used to describe a Pick theorem for any bounded region in $\mathbb{C}^{d}$.

\section{INTRODUCTION}

Suppose $z_{1}, \ldots, z_{n}$ are distinct points in the complex open disk $\mathbb{D}$ and $w_{1}, \ldots, w_{n}$ are complex numbers. The classic Pick interpolation theorem for $\mathbb{D}$ states the following: there is a holomorphic function $f$ on $\mathbb{D}$ satisfying $f\left(z_{i}\right)=w_{i}$ for $i=1, \ldots, n$ and $\sup \{|f(z)|: z \in \mathbb{D}\} \leq 1$ if and only if the matrix $\left[\left(1-w_{i} \overline{w_{j}}\right)\left(1-z_{i} \overline{z_{j}}\right)^{-1}\right]$ is positive semidefinite.

It is well known that, in general, the analogue of Pick's theorem does not hold for domains other than the disk. The single matrix present in Pick's theorem is typically replaced with an infinite family of matrices. Abrahamse's interpolation theorem for multiply connected regions [1] was the first appearance of this phenomenon, where the family of matrices is naturally parametrized by a torus. Cole, Lewis and Wermer [7] approached the Pick problem in substantial generality by considering the problem for any uniform algebra. They showed that a solution exists when the Pick matrices associated to a large class of measures are all positive semidefinite. In [2], Agler and McCarthy carried out a deep analysis of the Pick problem for the bidisk. Though an infinite family of Pick matrices once again appeared, their simultaneous positivity was reformulated as a factorization problem for $H^{\infty}\left(\mathbb{D}^{2}\right)$. These results also hold for the polydisk, but the associated Pick theorem is not given in terms of the $H^{\infty}\left(\mathbb{D}^{d}\right)$ norm. See [3] and the references therein for a detailed treatment of all of these results.

In this paper, we present two Pick-type theorems in a multivariable setting. For $d \geq 2$, the open unit ball and polydisk in $\mathbb{C}^{d}$ will be denoted by $\mathbb{B}_{d}$ and $\mathbb{D}^{d}$, respectively. The Hardy space $H^{2}(\Omega)$ is defined as the closure of the multivariable analytic polynomials in $L^{2}(\partial \Omega, \theta)$, where $\partial \Omega$ is the distinguished boundary of $\Omega$ (i.e. $\partial \mathbb{D}^{d}=\mathbb{T}^{d}$ ) and $\theta$ is Lebesgue measure on $\partial \Omega$. The algebra of bounded analytic functions on $\Omega$ will be denoted by $H^{\infty}(\Omega)$. In Theorem 1.1 below, a Pick theorem for the polydisk and unit ball is obtained.

For an arbitrary bounded domain $\Omega \subset \mathbb{C}^{d}$ and Lebesgue measure $\mu$ on $\Omega$, the Bergman space $L_{a}^{2}(\Omega)$ is defined as those functions which are analytic on $\Omega$ and contained in $L^{2}(\Omega, \mu)$. In Theorem 1.2, a Pick result for any bounded domain in

Received by the editors October 5, 2011.

2010 Mathematics Subject Classification. Primary 47A57; Secondary 30E05, 46E22.

Key words and phrases. Pick interpolation, reproducing kernel, dual algebras.

This work was partially supported by an NSERC graduate scholarship. 
$\mathbb{C}^{d}$ is established using Bergman spaces. As is the case with Theorem 1.1 the associated Pick matrices arise from absolutely continuous measures on $\Omega$. These results are also valid for any weak*-closed subalgebra of $H^{\infty}(\Omega)$, a feature which is typically absent in Pick interpolation results. In both theorems, the symbol $k^{\nu}$ refers to a reproducing kernel function described in Section 2

Theorem 1.1. Suppose $\Omega$ is either $\mathbb{D}^{d}$ or $\mathbb{B}_{d}$ and that $z_{1}, \ldots, z_{n} \in \Omega$ and $w_{1}, \ldots$, $w_{n} \in \mathbb{C}$. Let $\mathcal{A}$ be any weak $k^{*}$-closed subalgebra of $H^{\infty}(\Omega)$. There is a function $\varphi \in \mathcal{A}$ with $\sup _{z \in \Omega}|\varphi(z)| \leq 1$ and $\varphi\left(z_{i}\right)=w_{i}$ for $i=1, \ldots, n$ if and only if the matrix

$$
\left[\left(1-w_{i} \overline{w_{j}}\right) k^{\nu}\left(z_{i}, z_{j}\right)\right]_{i, j=1}^{n} \geq 0
$$

is positive semidefinite for every measure of the form $\nu=|f|^{2} \theta$, where $\theta$ is Lebesgue measure on $\partial \Omega$ and $f \in H^{2}(\Omega)$.

Theorem 1.2. Suppose $\Omega$ is a bounded domain in $\mathbb{C}^{d}$ and that $z_{1}, \ldots, z_{n} \in \Omega$ and $w_{1}, \ldots, w_{n} \in \mathbb{C}$. Let $\mathcal{A}$ be any weak*-closed subalgebra of $H^{\infty}(\Omega)$. There is a function $\varphi \in \mathcal{A}$ with $\sup _{z \in \Omega}|\varphi(z)| \leq 1$ and $\varphi\left(z_{i}\right)=w_{i}$ for $i=1, \ldots, n$ if and only if the matrix

$$
\left[\left(1-w_{i} \overline{w_{j}}\right) k^{\nu}\left(z_{i}, z_{j}\right)\right]_{i, j=1}^{n} \geq 0
$$

is positive semidefinite for every measure of the form $\nu=|f|^{2} \mu$, where $\mu$ is Lebesgue measure on $\Omega$ and $f \in L_{a}^{2}(\Omega)$.

Theorem 1.1 and Theorem 1.2 both provide significant simplifications of the Cole-Lewis-Wermer approach for the algebra $H^{\infty}(\Omega)$ and its subalgebras. Even though an infinite family of kernel functions is still required, they are given a concrete description. These theorems will be established using some recent results of Davidson and the author [8], where a general framework for Pick-type theorems was developed using the theory of dual operator algebras and their preduals. Dual algebra techniques in Pick interpolation can also be seen in the paper of McCullough [11. McCullough retrieves Theorem 1.1 when $\mathcal{A}=H^{\infty}(\Omega)$ (Theorem 5.12, 11]). In this case, it is shown that the functions $f$ may be taken to be bounded and have strictly positive modulus on $\partial \Omega$.

In Section 2, we will briefly discuss reproducing kernel Hilbert spaces and their multipliers. A natural notion of equivalence between reproducing kernel Hilbert spaces is introduced and applied to cyclic subspaces of $H^{2}(\Omega)$ and $L_{a}^{2}(\Omega)$ in Theorem 2.6 In order to prove the desired results, some dual algebra results of BercoviciWestwood [6] and Prunaru [12 are invoked, which, when combined with the other results in Section 2, establish the desired results.

\section{Reproducing Kernel Hilbert sPaCes AND their MUltipliers}

We say that a Hilbert space $H$ of $\mathbb{C}$-valued functions on $X$ is a reproducing kernel Hilbert space if point evaluations are continuous. For every $x \in X$, the reproducing kernel at $x$ is the function $k_{x} \in H$ which satisfies $f(x)=\left\langle f, k_{x}\right\rangle$ for $f \in H$. The associated positive definite kernel on $X \times X$ is given by $k(x, y):=$ $\left\langle k_{y}, k_{x}\right\rangle$. A multiplier $\varphi$ of $H$ is a function on $X$ such that $\varphi f \in H$ for every $f \in H$. Each multiplier $\varphi$ induces a bounded multiplication operator $M_{\varphi}$ on $H$. The multiplier algebra of $H$, denoted $M(H)$, is the operator algebra consisting of all such $M_{\varphi}$. The multiplier algebra is unital, maximal abelian and closed in the 
weak operator topology. The adjoints of multiplication operators are characterized by the fundamental identity $M_{\varphi}^{*} k_{x}=\overline{\varphi(x)} k_{x}$.

Example 2.1. The kernel functions for the Hardy spaces $H^{2}\left(\mathbb{D}^{d}\right)$ and $H^{2}\left(\mathbb{B}_{d}\right)$ are given by

$$
k^{H^{2}\left(\mathbb{D}^{d}\right)}(z, w)=\prod_{k=1}^{d} \frac{1}{1-z_{i} \overline{w_{i}}} \text { and } k^{H^{2}\left(\mathbb{B}_{d}\right)}(z, w)=\frac{1}{\left(1-\langle z, w\rangle_{\mathbb{C}^{d}}\right)^{d}},
$$

respectively. When $\Omega$ is either $\mathbb{D}^{d}$ or $\mathbb{B}_{d}$, the multiplier algebra $M\left(H^{2}(\Omega)\right)$ is equal to $H^{\infty}(\Omega)$, and this identification is isometric: $\left\|M_{\varphi}\right\|=\|\varphi\|_{\infty}$ for $\varphi \in H^{\infty}(\Omega)$. If $\Omega$ is any bounded domain in $\mathbb{C}^{d}$, the kernel function for the Bergman spaces $L_{a}^{2}(\Omega)$ is generally difficult to compute. Some familiar examples are given by

$$
k^{L_{a}^{2}\left(\mathbb{D}^{d}\right)}(z, w)=\prod_{k=1}^{d} \frac{1}{\left(1-z_{i} \overline{w_{i}}\right)^{2}} \text { and } k^{L_{a}^{2}\left(\mathbb{B}_{d}\right)}(z, w)=\frac{1}{\left(1-\langle z, w\rangle_{\mathbb{C}^{d}}\right)^{d+1}} .
$$

It is easy to verify that $M\left(L_{a}^{2}(\Omega)\right)=H^{\infty}(\Omega)$ and that $\left\|M_{\varphi}\right\|=\|\varphi\|_{\infty}$ for any $\varphi \in H^{\infty}(\Omega)$. See the book of Krantz [10] for a detailed treatment of these spaces.

This note will concern itself only with reproducing kernel Hilbert spaces of analytic functions. We further assume that $H$ is endowed with an $L^{2}$ norm; i.e., there is some set $\Delta$ along with a $\sigma$-algebra of subsets and a measure $\mu$ such that $H$ is a closed subspace of $L^{2}(\Delta, \mu)$. The set $\Delta$ may play different roles depending on the context. For the Hardy space of the polydisk or unit ball, $\Delta$ is taken to be either $\mathbb{T}^{d}$ or $\partial \mathbb{B}_{d}$, respectively, and $\mu$ is the corresponding Lebesgue measure on these sets. For the Bergman space $L_{a}^{2}(\Omega)$, we simply take $\Delta=\Omega$ and $\mu$ to be Lebesgue measure on $\Omega$. We also assume that $H$ contains the constant function 1 , so that every multiplier of $H$ is contained in $H$.

Given an algebra of multipliers $\mathcal{A}$ on $H$ and a measure $\nu$ on $\Delta$, let $\mathcal{A}^{2}(\nu)$ denote the closure of $\mathcal{A}$ in $L^{2}(\Delta, \nu)$. The measure $\nu$ is said to be dominating for $X$ (with respect to $\mathcal{A}$ ) if $\mathcal{A}^{2}(\nu)$ is a reproducing kernel Hilbert space on $X$. We will write $k_{x}^{A^{2}(\nu)}$ for the reproducing kernel on this space or more simply as $k_{x}^{\nu}$ when the context is clear. The associated positive definite kernel function on $X \times X$ will be denoted by $k^{\nu}(x, y):=\left\langle k_{y}^{\nu}, k_{x}^{\nu}\right\rangle_{A^{2}(\nu)}$. For any such $\nu, \mathcal{A}$ is obviously an algebra of multipliers on $\mathcal{A}^{2}(\nu)$.

Remark 2.2. Our notion of a dominating measure differs slightly from Cole-LewisWermer 7. In their setting, $\mathcal{A}$ is a uniform algebra and $\Delta$ is the maximal idea space of $\mathcal{A}$. A measure $\mu$ on $\Delta$ is said to be dominating for a subset $\Lambda$ of $\Delta$ if there is a constant $C$ such that $|\varphi(\lambda)| \leq C\|\varphi\|_{L^{2}(\mu)}$ for every $\lambda \in \Lambda$. Their theorem solves the so-called weak Pick problem for $\mathcal{A}$ : Given $\epsilon>0, w_{1}, \ldots, w_{n} \in \mathbb{C}$ and $\lambda_{1}, \ldots, \lambda_{n} \in \Delta$, there is a function $\varphi \in \mathcal{A}$ with $\varphi\left(\lambda_{i}\right)=w_{i}$ for $i=1, \ldots, n$ and $\|\varphi\|<1+\epsilon$ if and only if

$$
\left[\left(1-w_{i} \overline{w_{j}}\right) k^{\mu}\left(\lambda_{i}, \lambda_{j}\right)\right] \geq 0
$$

for every measure $\mu$ which is dominating for $\left\{\lambda_{1}, \ldots, \lambda_{n}\right\}$.

A unital, weak-* closed subalgebra of $B(H)$ will be called a dual algebra. The predual $\mathcal{A}_{*}$ of a dual algebra may be identified canonically with a quotient of the 
trace class operators on $H$. A dual algebra $\mathcal{A}$ is said to have property $\mathbb{A}_{1}(1)$ if every $\pi \in \mathcal{A}_{*}$ with $\|\pi\|<1$ may be written as

$$
\pi(A)=\langle A x, y\rangle_{H}
$$

for some $x, y \in H$ which satisfy $\|x\|\|y\|<1$. See the manuscript of Bercovici, Foias and Pearcy [5] for a detailed treatment of dual algebras and the structure of their preduals.

If $H$ is a reproducing kernel Hilbert space and $\mathcal{A} \subset M(H)$ is a dual algebra, we call $\mathcal{A}$ a dual algebra of multipliers. If $L$ is an invariant subspace of $\mathcal{A}$, then $L$ is also a reproducing kernel Hilbert space with the kernel function at $x$ given by $P_{L} k_{x}$. Clearly $\mathcal{A}$ is also a dual algebra of multipliers on $L$, and we denote the multiplication operator associated to $\varphi$ as $M_{f}^{L}$. It follows that $\left(M_{\varphi}^{L}\right)^{*} P_{L} k_{x}=\overline{\varphi(x)} P_{L} k_{x}$ for $x \in X$. Note that if $f(x)=0$ for every $f \in L$, then $P_{L} k_{x}=0$. Since we are principally concerned with the evaluation of multipliers, it is useful to extend the kernel function for $L$ to points which are annihilated by every function in $L$. If $f \in H$, we denote the closed cyclic subspace generated by $\mathcal{A}$ and $f$ as $\mathcal{A}[f]$. The following lemma appears as Lemma 2.1 in 8 .

Lemma 2.3. Suppose $\mathcal{A}$ is a dual algebra of multipliers on a reproducing kernel Hilbert space $H$ and let $\mathcal{I}_{x}$ denote the ideal of functions in $\mathcal{A}$ which vanish on $x$. If $f \in H$ and $\mathcal{A}[f] \neq I_{x}[f]$, then the one-dimensional subspace $\mathcal{A}[f] \ominus I_{x}[f]$ contains a nonzero vector $k_{x}^{f}$ such that $P_{\mathcal{A}[f]} k_{x}=k_{x}^{f}$ if $f(x) \neq 0$ and $\left(M_{\varphi}^{f}\right)^{*} k_{x}^{f}=\overline{\varphi(x)} k_{x}^{f}$.

If $L=\mathcal{A}[f]$ for $f \in H$, we will use the notation $k_{x}^{f}$ and $M_{\varphi}^{f}$ for $k_{x}^{L}$ and $M_{\varphi}^{L}$, respectively. The following result of Davidson and the author ( $[$, Theorem 3.4) gives a Pick theorem for dual algebras of multipliers which have property $\mathbb{A}_{1}(1)$.

Theorem 2.4. Suppose that $H$ is both a reproducing kernel Hilbert space over a set $X$ and that $\mathcal{A}$ is a dual algebra of multipliers on $H$ which has property $\mathbb{A}_{1}(1)$. Then the following statement holds: given $x_{1}, \ldots, x_{n} \in X$ and $w_{1}, \ldots, w_{n} \in \mathbb{C}$, there is a multiplier $\varphi \in \mathcal{A}$ such that $\varphi\left(x_{i}\right)=w_{i}$ and $\left\|M_{\varphi}\right\| \leq 1$ if and only if the matrices

$$
\left[\left(1-w_{i} \overline{w_{j}}\right)\left\langle k_{x_{j}}^{f}, k_{x_{i}}^{f}\right\rangle\right]
$$

are positive semidefinite for every $f \in H$.

When $H$ is contained in an ambient $L^{2}$ space, we seek a nicer description of the cyclic subspaces $\mathcal{A}[f]$. Suppose $H$ and $K$ are reproducing kernel Hilbert spaces on $X$ with kernels $k$ and $j$, respectively, and that $U: H \rightarrow K$ is a unitary map. We say that $U$ is a reproducing kernel Hilbert space isomorphism if for every $x \in X$ there is a nonzero scalar $c_{x}$ such that $U k_{x}=c_{x} j_{x}$. We require the following easy proposition, the details of which may be found in ([3, Section 2.6).

Proposition 2.5. Suppose $H$ and $K$ are reproducing kernel Hilbert spaces on a set $X$ and that $U: H \rightarrow K$ is a reproducing kernel Hilbert space isomorphism. As sets, the multiplier algebras of $H$ and $K$ are equal. Moreover, the map $U$ induces a unitary equivalence between $M(H)$ and $M(K)$.

The following result shows that cyclic subspaces may naturally be identified with $H$ under a different norm. Douglas and Sarkar obtain a similar result (Lemma 2, 9]) in the language of Hilbert modules (though we require slightly more information here). Recall that if $H \subset L^{2}(\Delta, \mu), \mathcal{A} \subset M(H)$ and $\nu$ is some measure on $\Delta$, then $\mathcal{A}^{2}(\nu)$ is the closure of $\mathcal{A}$ in $L^{2}(\Delta, \nu)$. 
Theorem 2.6. Suppose $H$ is a reproducing kernel Hilbert space of analytic functions on some bounded domain $\Omega$. Suppose further that there is a measure space $(\Delta, \mu)$ such that $H$ is a closed subspace of $L^{2}(\Delta, \mu)$. If $\mathcal{A}$ is any dual algebra of multipliers on $H$, then $\mathcal{A}^{2}\left(|f|^{2} \mu\right)$ is a reproducing kernel Hilbert space on the set $\Omega_{f}:=\left\{z \in \Omega:\left\langle f, k_{z}^{f}\right\rangle_{H} \neq 0\right\}$. The reproducing kernel for $\mathcal{A}^{2}\left(|f|^{2} \mu\right)$ is given by

$$
j^{f}(z, w)=\frac{k^{f}(z, w)}{\left\langle f, k_{z}^{f}\right\rangle_{H}{\overline{\left\langle f, k_{w}^{L}\right\rangle_{H}}}} .
$$

Moreover, there is a reproducing kernel Hilbert space isomorphism

$$
U: \mathcal{A}[f] \rightarrow \mathcal{A}^{2}\left(|f|^{2} \mu\right) \text {. }
$$

In particular, any measure of the form $|f|^{2} \mu$ is dominating for $\Omega_{f}$ with respect to $\mathcal{A}$.

Proof. Define a linear map $V: \mathcal{A} \rightarrow \mathcal{A}[f]$ by $V \varphi=\varphi f$. It is clear by the definition of the norms involved that $V$ extends to a unitary

$$
V: \mathcal{A}^{2}\left(|f|^{2} \mu\right) \rightarrow \mathcal{A}[f] .
$$

We claim that $U:=V^{*}$ is the required isomorphism. For notational convenience, let $\mathcal{A}^{2}:=\mathcal{A}^{2}\left(|f|^{2} \mu\right)$. If $\varphi \in \mathcal{A}$ and $z \in \Omega_{f}$, we have

$$
\left\langle\varphi, V^{*} k_{z}^{f}\right\rangle_{A^{2}}=\left\langle\varphi f, k_{z}^{f}\right\rangle_{H}=\left\langle f,\left(M_{\varphi}^{f}\right)^{*} k_{z}^{f}\right\rangle_{H}=\varphi(z)\left\langle f, k_{z}^{f}\right\rangle_{H}
$$

By assumption, $\left\langle f, k_{z}^{f}\right\rangle_{H} \neq 0$, and so the vector ${\overline{\left\langle f, k_{z}^{f}\right\rangle_{H}}}_{H}^{-1} V^{*} k_{z}^{f}$ is the reproducing kernel at the point $z$ for any function in $\mathcal{A}$. In order to show this for any function $\varphi \in \mathcal{A}^{2}$, find $\varphi_{n} \in \mathcal{A}$ with $\left\|\varphi_{n}-\varphi\right\|_{\mathcal{A}^{2}}$ tending to 0 . Since $\varphi \in L^{2}\left(\Delta,|f|^{2} \mu\right)$, it follows that $\varphi f \in L^{2}(\Delta, \mu)$. This in turn implies that the sequence $\left\{\varphi_{n} f\right\}$ is Cauchy in $L^{2}(\Delta, \mu)$ and that its limit must be $\varphi f$. The subspace $\mathcal{A}[f]$ is closed, and so $\varphi f \in \mathcal{A}[f]$. Taking an inner product against $k_{z}^{f}$ implies that $\varphi_{n}(z) \rightarrow \varphi(z)$ for any $z \in \Omega_{f}$. Consequently

$$
\begin{aligned}
\left\langle\varphi, \frac{V^{*} k_{z}^{f}}{\overline{\left\langle f, k_{z}^{f}\right\rangle_{H}}}\right\rangle_{\mathcal{A}^{2}} & =\left\langle\varphi f, \frac{k_{z}^{f}}{\overline{\left\langle f, k_{z}^{f}\right\rangle_{H}}}\right\rangle_{H} \\
& =\lim _{n \rightarrow \infty}\left\langle\varphi_{n} f, \frac{k_{z}^{f}}{\overline{\left\langle f, k_{z}^{f}\right\rangle_{H}}}\right\rangle_{H}=\lim _{n \rightarrow \infty} \varphi_{n}(z)=\varphi(z) .
\end{aligned}
$$

Now set $j_{z}^{f}:=\left(\overline{\left\langle f, k_{z}^{f}\right\rangle_{H}}\right)^{-1} V^{*} k_{z}^{f}$. The above reasoning shows that $j_{z}^{f}$ is the reproducing kernel for $\mathcal{A}^{2}$, and

$$
j^{f}(z, w):=\left\langle j_{w}^{f}, j_{z}^{f}\right\rangle_{\mathcal{A}^{2}}=\frac{\left\langle V^{*} k_{w}^{f}, V^{*} k_{v}^{f}\right\rangle_{\mathcal{A}^{2}}}{\left\langle f, k_{z}^{f}\right\rangle_{H} \overline{\left\langle f, k_{w}^{f}\right\rangle_{H}}}=\frac{k^{f}(z, w)}{\left\langle f, k_{z}^{f}\right\rangle_{L} \overline{\left\langle f, k_{w}^{f}\right\rangle_{H}}},
$$

which proves the theorem.

Corollary 2.7. Suppose $\mathcal{A}$ and $H$ satisfy the hypotheses of Theorem 2.6. Then the matrix

$$
\left[\left(1-w_{i} \overline{w_{j}}\right) k^{f}\left(z_{i}, z_{j}\right)\right]_{i, j=1}^{n}
$$

is positive semidefinite if and only if

$$
\left[\left(1-w_{i} \overline{w_{j}}\right) j^{f}\left(z_{i}, z_{j}\right)\right]_{i, j=1}^{n}
$$

is positive semidefinite. 
Proof. By Theorem 2.6, the matrix $\left[\left(1-w_{i} \overline{w_{j}}\right) j^{f}\left(z_{i}, z_{j}\right)\right]$ is the Schur product of $\left[\left(1-w_{i} \overline{w_{j}}\right) k^{f}\left(z_{i}, z_{j}\right)\right]$ and $\left[\left(\left\langle f, k_{z_{j}}^{f}\right\rangle \overline{\left\langle f, k_{z_{i}}^{f}\right\rangle}\right)^{-1}\right]$, the latter of which is manifestly positive semidefinite.

We can now summarize the results of this section so far.

Theorem 2.8. Let $(\Delta, \mathcal{B}, \mu)$ be a $\sigma$-finite measure space such that $H$ is both a reproducing kernel Hilbert space over a set $X$ and a closed subspace of $L^{2}(\Delta, \mu)$. Suppose that $\mathcal{A}$ is a dual algebra of multipliers on $H$ which has property $\mathbb{A}_{1}(1)$. Then the following statement holds: given $x_{1}, \ldots, x_{n} \in X$ and $w_{1}, \ldots, w_{n} \in \mathbb{C}$, there is a multiplier $\varphi \in \mathcal{A}$ such that $\varphi\left(x_{i}\right)=w_{i}$ and $\left\|M_{\varphi}\right\| \leq 1$ if and only if the matrix

$$
\left[\left(1-w_{i} \overline{w_{j}}\right) k^{\nu}\left(x_{i}, x_{j}\right)\right]_{i, j=1}^{n}
$$

is positive semidefinite for every measure of the form $\nu=|f|^{2} \mu$, where $f \in H$.

Proof. If such a $\varphi$ exists, then $M_{\varphi}^{f}$ is a contraction for every $f \in H$. It follows that the operator

$$
I-M_{\varphi}^{f}\left(M_{\varphi}^{f}\right)^{*}
$$

is positive semidefinite. Taking an inner product against finite spans of the functions $k_{z}^{f}$ implies that the matrices

$$
\left[\left(1-w_{i} \overline{w_{j}}\right) k^{f}\left(z_{i}, z_{j}\right)\right]_{i, j=1}^{n}
$$

are positive semidefinite. Now apply Corollary 2.7 and note that $k^{|f|^{2} \mu}=j^{f}$. The nontrivial direction is obtained by combining Theorem 2.4 and Corollary 2.7 .

We will use the following important factorization result of Bercovici-Westwood ([6], Theorem 1). Recall that $\theta$ is Lebesgue measure on $\partial \Omega$.

Theorem 2.9 (Bercovici-Westwood). Suppose $\Omega$ is either $\mathbb{D}^{d}$ or $\mathbb{B}_{d}$. Then for any function $h \in L^{1}(\partial \Omega, \theta)$ and $\varepsilon>0$, there are functions $f$ and $g$ in $H^{2}(\Omega)$ such that $\|f\|_{2}\|g\|_{2} \leq\|h\|_{1}$ and $\|f-g \bar{h}\|_{2}<\varepsilon$. In particular, $\mathcal{M}\left(H^{2}(\Omega)\right)$ has property $\mathbb{A}_{1}(1)$.

Property $\mathbb{A}_{1}(1)$ is hereditary for weak-* closed subspaces ([5], Proposition 2.04), and so we may combine the above result with Theorem 2.8, which proves Theorem 1.1. In order to prove Theorem 1.2, we employ a versatile factorization theorem of Prunaru (12, Theorem 4.1). This result applies to any instance where $H$ is a reproducing kernel Hilbert space on a measure space $(X, \mathcal{B}, \mu)$ and $H$ is a closed subspace of $L^{2}(X, \mu)$. In particular, it applies to any Bergman space, but not to Hardy space. Any reproducing kernel Hilbert space which satisfies these hypotheses always has the property that $\left\|M_{\varphi}\right\|=\sup _{x \in X}|\varphi(x)|$ for any multiplier $\varphi$.

Theorem 2.10 (Prunaru). Let $(X, \mathcal{B}, \mu)$ be a $\sigma$-finite measure space such that $H$ is a reproducing kernel Hilbert space over $X$ and a closed subspace of $L^{2}(X, \mu)$. Then the multiplier algebra $\mathcal{M}(H)$ has property $\mathbb{A}_{1}(1)$.

In fact, Prunaru shows that $M(H)$ satisfies a much stronger predual factorization property known as $X(0,1)$. In the particular case where $H$ is a Bergman space, Bercovici proves a result similar to Theorem 2.10 in [4]. An application of Theorem 2.8 gives us what we need. 
Corollary 2.11. Let $(X, \mathcal{B}, \mu)$ be a $\sigma$-finite measure space such that $H$ is both a reproducing kernel Hilbert space over $X$ and a closed subspace of $L^{2}(X, \mu)$. Suppose $\mathcal{A}$ is a dual algebra of multipliers on $H$. If $x_{1}, \ldots, x_{n} \in X$ and $w_{1}, \ldots, w_{n} \in \mathbb{C}$, then there is a function $\varphi \in \mathcal{A}$ with $\|\varphi\|_{\infty} \leq 1$ and $\varphi\left(x_{i}\right)=w_{i}$ for $i=1, \ldots, n$ if and only if

$$
\left[\left(1-w_{i} \overline{w_{j}}\right) k^{\nu}\left(x_{i}, x_{j}\right)\right]_{i, j=1}^{n} \geq 0
$$

for every measure of the form $\nu=|f|^{2} \mu$, for $f \in H$.

Theorem 1.2 now follows from Corollary 2.11 by taking $X$ to be a bounded domain $\Omega$ and setting $H=L_{a}^{2}(\Omega)$.

\section{ACKNOWLEDGEMENTS}

The author wishes to express his gratitude to K. R. Davidson and J. E. McCarthy for many helpful discussions and comments on this manuscript.

\section{REFERENCES}

[1] M.B. Abrahamse, The Pick interpolation theorem for finitely connected domains, Michigan Math. J. 26 (1979), 195-203. MR532320 (80j:30052)

[2] J. Agler and J.E. McCarthy, Nevanlinna-Pick interpolation on the bidisk, J. Reine Angew. Math. 506 (1999), 191-204. MR1665697 (2000a:47034)

[3] J. Agler and J.E. McCarthy, Pick interpolation and Hilbert function spaces, Graduate Studies in Mathematics 44, Amer. Math. Soc., Providence, RI, 2002. MR 1882259 (2003b:47001)

[4] H. Bercovici, The algebra of multiplication operators on Bergman spaces, Arch. Math. (Basel) 48 (1987), 165-175. MR878428 (88e:47057)

[5] H. Bercovici, C. Foiaş, and C. Pearcy, Dual algebras with applications to invariant subspaces and dilation theory, CBMS Regional Conference Series in Mathematics, 56, Amer. Math. Soc., Providence, RI, 1985. MR787041 (87g:47091)

[6] H. Bercovici and D. Westwood, The factorization of functions in the polydisc, Houston J. Math. 18 (1992), No. 1, 1-6. MR.1159434 (93b:32006)

[7] B.J. Cole, K. Lewis, and J. Wermer, Pick conditions on a uniform algebra and von Neumann inequalities, J. Funct. Anal. 107 (1992), 235-254. MR.1172022 (93e:46059)

[8] K.R. Davidson and R. Hamilton, Nevanlinna-Pick interpolation and factorization of linear functionals, Integral Equations Operator Theory 70 (2011), No. 1, 125-149. MR2786738 (2012e:47045)

[9] R.G. Douglas and J. Sarkar, Some remarks on the Toeplitz corona problem, Hilbert spaces of analytic functions, CRM Proc. Lecture Notes, 51, 81-89, Amer. Math. Soc., Providence, RI, 2010. MR2648868 (2011i:46051)

[10] S.G. Krantz, Function theory of several complex variables, The Wadsworth \& Brooks/Cole Mathematics Series, Second Edition, Pacific Grove, CA, 1992. MR1162310 (93c:32001)

[11] S. McCullough, Nevanlinna-Pick type interpolation in a dual algebra, J. Funct. Anal. 135 (1996), No. 1, 93-131. MR.1367626 (96j:47013)

[12] B. Prunaru, A factorization theorem for multiplier algebras of reproducing kernel Hilbert spaces, Canadian Math. Bull., to appear.

Department of Pure Mathematics, University of Waterloo, 200 University Avenue West, Waterloo, Ontario N2L-3G1, Canada

E-mail address: rhamilto@uwaterloo.ca 